\title{
End-of-Life Care: Questions and Answers
}

When a patient's health care team determines that the cancer can no longer be controlled, medical testing and cancer treatment often stop. But the patient's care continues. The care focuses on making the patient comfortable. The patient receives medications and treatments to control pain and other symptoms, such as constipation, nausea, and shortness of breath. Some patients remain at home during this time, while others enter a hospital or other facility. Either way, services are available to help patients and their families with the medical, psychological, and spiritual issues surrounding dying. A hospice often provides such services.

The time at the end of life is different for each person. Each individual has unique needs for information and support. The patient's and family's questions and concerns about the end of life should be discussed with the health care team as they arise.

The following information can help answer some of the questions that many patients, their family members, and caregivers have about the end of life.

\section{How long is the patient expected to live?}

Patients and their family members often want to know how long a person is expected to live. This is a hard question to answer. Factors such as the where the cancer is located and whether the patient has other illnesses can affect what will happen. Although doctors may be able to make an estimate based on what they know about the patient, they might be hesitant to do so. Doctors may be concerned about over- or under-estimating the patient's life span. They also might be fearful of instilling false hope or destroying a person's hope.

\section{When caring for the patient at home, when should the caregiver call for professional} help?

When caring for a patient at home, there may be times when the caregiver needs assistance from the patient's health care team. A caregiver can contact the patient's doctor or nurse for help in any of the following situations:

- The patient is in pain that is not relieved by the prescribed dose of pain medication;

- The patient shows discomfort, such as grimacing or moaning; 
- The patient is having trouble breathing and seems upset;

- The patient is unable to urinate or empty the bowels;

- The patient has fallen;

- The patient is very depressed or talking about committing suicide;

- The caregiver has difficulty giving medication to the patient;

- The caregiver is overwhelmed by caring for the patient, or is too grieved or afraid to be with the patient; or

- At any time the caregiver does not know how to handle a situation.

3. What are some ways that caregivers can provide emotional comfort to the patient?

Everyone has different needs, but some emotions are common to most dying patients. These include fear of abandonment and fear of being a burden. They also have concerns about loss of dignity and loss of control. Some ways caregivers can provide comfort are as follows:

- Keep the person company — talk, watch movies, read, or just be with the person.

- Allow the person to express fears and concerns about dying, such as leaving family and friends behind. Be prepared to listen.

- Be willing to reminisce about the person's life.

- Avoid withholding difficult information. Most patients prefer to be included in discussions about issues that concern them.

- Reassure the patient that you will honor advance directives, such as living wills.

- Ask if there is anything you can do.

- Respect the person's need for privacy.

4. What are the signs that death is approaching? What can the caregiver do to make the patient comfortable?

Certain signs and symptoms can help a caregiver anticipate when death is near. They are described below, along with suggestions for managing them. It is important to remember that not every patient experiences each of the signs and symptoms. In addition, the presence of one or more of these symptoms does not necessarily indicate that the patient is close to death. A member of the patient's health care team can give family members and caregivers more information about what to expect.

- Drowsiness, increased sleep, and/or unresponsiveness (caused by changes in the patient's metabolism).

The caregiver and family members can plan visits and activities for times when the patient is alert. It is important to speak directly to the patient and talk as if the person can hear, even if there is no response. Most patients are still able to hear after they are no longer able to speak. Patients should not be shaken if they do not respond. 
- Confusion about time, place, and/or identity of loved ones; restlessness; visions of people and places that are not present; pulling at bed linens or clothing (caused in part by changes in the patient's metabolism). Gently remind the patient of the time, date, and people who are with them. If the patient is agitated, do not attempt to restrain the patient. Be calm and reassuring. Speaking calmly may help to re-orient the patient.

- Decreased socialization and withdrawal (caused by decreased oxygen to the brain, decreased blood flow, and mental preparation for dying).

Speak to the patient directly. Let the patient know you are there for them. The patient may be aware and able to hear, but unable to respond. Professionals advise that giving the patient permission to "let go" can be helpful.

- Decreased need for food and fluids, and loss of appetite (caused by the body's need to conserve energy and its decreasing ability to use food and fluids properly).

Allow the patient to choose if and when to eat or drink. Ice chips, water, or juice may be refreshing if the patient can swallow. Keep the patient's mouth and lips moist with products such as glycerin swabs and lip balm.

- Loss of bladder or bowel control (caused by the relaxing of muscles in the pelvic area).

Keep the patient as clean, dry, and comfortable as possible. Place disposable pads on the bed beneath the patient and remove them when they become soiled.

- Darkened urine or decreased amount of urine (caused by slowing of kidney function and/or decreased fluid intake).

Caregivers can consult a member of the patient's health care team about the need to insert a catheter to avoid blockage. A member of the health care team can teach the caregiver how to take care of the catheter if one is needed.

- Skin becomes cool to the touch, particularly the hands and feet; skin may become bluish in color, especially on the underside of the body (caused by decreased circulation to the extremities).

Blankets can be used to warm the patient. Although the skin may be cool, patients are usually not aware of feeling cold. Caregivers should avoid warming the patient with electric blankets or heating pads, which can cause burns.

- Rattling or gurgling sounds while breathing, which may be loud; breathing that is irregular and shallow; decreased number of breaths per minute; breathing that alternates between rapid and slow (caused by congestion from decreased fluid 
consumption, a buildup of waste products in the body, and/or a decrease in circulation to the organs).

Breathing may be easier if the patient's body is turned to the side and pillows are placed beneath the head and behind the back. Although labored breathing can sound very distressing to the caregiver, gurgling and rattling sounds do not cause discomfort to the patient. An external source of oxygen may benefit some patients. If the patient is able to swallow, ice chips also may help. In addition, a cool mist humidifier may help make the patient's breathing more comfortable.

- Turning the head toward a light source (caused by decreasing vision).

Leave soft, indirect lights on in the room.

- Increased difficulty controlling pain (caused by progression of the disease).

It is important to provide pain medications as the patient's doctor has prescribed. The caregiver should contact the doctor if the prescribed dose does not seem adequate. With the help of the health care team, caregivers can also explore methods such as massage and relaxation techniques to help with pain.

- Involuntary movements (called myoclonus), changes in heart rate, and loss of reflexes in the legs and arms are additional signs that the end of life is near.

\section{What are the signs that the patient has died?}

- There is no breathing or pulse.

- The eyes do not move or blink, and the pupils are dilated (enlarged). The eyelids may be slightly open.

- The jaw is relaxed and the mouth is slightly open.

- The body releases the bowel and bladder contents.

- The patient does not respond to being touched or spoken to.

\section{What needs to be done after the patient has died?}

After the patient has passed away, there is no need to hurry with arrangements. Family members and caregivers may wish to sit with the patient, talk, or pray. When the family is ready, the following steps can be taken.

- Place the body on its back with one pillow under the head. If necessary, caregivers or family members may wish to put the patient's dentures or other artificial parts in place. 
- If the patient is in a hospice program, follow the guidelines provided by the program. A caregiver or family member can request a hospice nurse to verify the patient's death.

- Contact the appropriate authorities in accordance with local regulations. If the patient has requested not to be resuscitated through a Do-Not-Resuscitate (DNR) order or other mechanism, do not call 911.

- Contact the patient's doctor and funeral home.

- When the patient's family is ready, call other family members, friends, and clergy.

- Provide or obtain emotional support for family members and friends to cope with their loss.

\section{What additional resources offer information about end-of-life issues?}

The following National Cancer Institute (NCI) resources are available by calling the Cancer Information Service (CIS) (see below) at 1-800-4-CANCER (1-800-422-6237). They can also be accessed on the NCI's Cancer.gov Web site at http://cancer.gov/cancer_information/coping/ by clicking on the title under "End-of-Life Issues.”

- $\quad$ The NCI fact sheet Hospice provides information about hospice care and includes contact information for hospice organizations.

- Advance Directives is an NCI fact sheet that discusses a patient's rights regarding medical treatment.

- The NCI fact sheet Home Care for Cancer Patients provides information and resources related to home care services.

- The NCI booklet Advanced Cancer: Living Each Day provides practical support to cancer patients, families, and friends.

- $\quad$ PDQ ${ }^{\circledR}$ supportive care summaries on loss, grief, and bereavement.

$$
\text { \# \# \# }
$$

\section{$\underline{\text { Sources of National Cancer Institute Information }}$}

\section{Cancer Information Service}

Toll-free: 1-800-4-CANCER (1-800-422-6237)

TTY (for deaf and hard of hearing callers): 1-800-332-8615 


\section{NCI Online}

Internet

Use http://cancer.gov to reach the NCI's Web site.

LiveHelp

Cancer Information Specialists offer online assistance through the LiveHelp link on the NCI's Web site.

This fact sheet was reviewed on 10/30/02 